\title{
On Second-Order Differential Equations with Nonsmooth Second Member
}

\author{
M. Milla Miranda, ${ }^{1}$ A. T. Lourêdo, ${ }^{1}$ and L. A. Medeiros $^{2}$ \\ ${ }^{1}$ DM, UEPB, Campina Grande-PB, Brazil \\ ${ }^{2}$ IM, UFRJ, Rio de Janeiro, RJ, Brazil \\ Correspondence should be addressed to M. Milla Miranda; milla@im.ufrj.br \\ Received 15 January 2014; Accepted 27 February 2014; Published 24 March 2014 \\ Academic Editors: A. Bellouquid and C. Join
}

Copyright (C) 2014 M. Milla Miranda et al. This is an open access article distributed under the Creative Commons Attribution License, which permits unrestricted use, distribution, and reproduction in any medium, provided the original work is properly cited.

\begin{abstract}
In an abstract framework, we consider the following initial value problem: $u^{\prime \prime}+\mu A u+F(u) u=f$ in $(0, T), u(0)=u^{0}, u^{\prime}(0)=u^{1}$, where $\mu$ is a positive function and $f$ a nonsmooth function. Given $u^{0}, u^{1}$, and $f$ we determine $F(u)$ in order to have a solution $u$ of the previous equation. We analyze two cases of $F(u)$. In our approach, we use the Theory of Linear Operators in Hilbert Spaces, the compactness Aubin-Lions Theorem, and an argument of Fixed Point. One of our two results provides an answer in a certain sense to an open question formulated by Lions in (1981, Page 284).
\end{abstract}

\section{Introduction}

Let $V$ and $H$ be two real separable Hilbert spaces with $V$ dense in $H$ and $V$ continuously embedding in $H$. The scalar product and norms of $V$ and $H$ are represented, respectively, by

$$
(u, v),|u|, \quad((u, v)),\|u\| .
$$

Let $A$ be the self-adjoint operator of $H$ defined by the triplet $\{V, H,((u, v))\}$. Consider $\alpha \in \mathbb{R}, \alpha \geq 0$. We denote by $D\left(A^{\alpha}\right)$ the Hilbert space

$$
D\left(A^{\alpha}\right)=\left\{u \in H ; A^{\alpha} u \in H\right\}
$$

equipped with the scalar product

$$
(u, v)_{D\left(A^{\alpha}\right)}=\left(A^{\alpha} u, A^{\alpha} v\right)
$$

(cf. Lions [1]).

Consider the following initial value problem:

$$
\begin{gathered}
u^{\prime \prime}+\mu A u+F(u) u=f \quad \text { in }(0, T), \\
u(0)=u^{0}, \quad u^{\prime}(0)=u^{1},
\end{gathered}
$$

where $\mu$ is a positive function and $f$ a nonsmooth function.
The objective of this work is to study the following inverse problem: given $u^{0}, u^{1}$, and $f \in L^{2}\left(0, T ; D\left(A^{\alpha}\right)^{\prime}\right)\left(D\left(A^{\alpha}\right)^{\prime}\right.$ dual space of $\left.D\left(A^{\alpha}\right)\right)$ to determine $F(u)$ such that Problem (4) has a solution $u$. We analyze two cases of $F(u)$, more precisely, the cases

$$
\begin{aligned}
& F(u)=\left|A^{-\theta / 2} u\right|^{2 p}, \\
& F(u)=\|u\|_{C^{0}([0, T] ; X)},
\end{aligned}
$$

where $X$ is an appropriate Hilbert space.

In Lions [2, Page 284], the following problem is formulated

$$
\begin{aligned}
& u^{\prime \prime}-\Delta u+\left[\int_{0}^{t}\left(\int_{\Omega} u^{2} d x\right) d s\right] u \\
& =v(t) \delta\left(x-x_{0}\right) \quad \text { in } \Omega \times(0, T), \\
& u=0 \quad \text { on } \Gamma \times(0, T), \\
& u(x, 0)=0, \quad u^{\prime}(x, 0)=0, \quad x \in \Omega,
\end{aligned}
$$

where $\Omega$ is an open bounded set of $\mathbb{R}^{n}$ with boundary $\Gamma$, $x_{0} \in \Omega$, and $\delta\left(x-x_{0}\right)$ being the Dirac mass supported at $\left\{x_{0}\right\}$. He says not to know if this problem admits a solution. 
He say also that one of the difficulties in the study of existence of solutions of the nonlinear equations lies in the difficulty in defining weak solutions, since the transposition method is essentially a linear method. This is ultimately connected to the fact that one cannot multiply distributions.

Problem (4) with $F(u)$ of the form (6) is an abstract formulation of Problem (7) with a slight modification of the nonlinear term. Theorem 3 gives the existence of solutions of this problem. In applications we give examples of Problem (7), with the modification of the nonlinear term, for $\Omega$ an open bounded set of $\mathbb{R}^{n}, n=1,2,3$.

In Grotta Ragazzo [3] the following equation is studied:

$$
u_{t t}-u_{x x}-a u+\left(\frac{1}{\pi} \int_{0}^{\pi} u^{2} d x\right)^{\alpha} u=0 \quad \text { in }(0, \pi) \times \mathbb{R} .
$$

This equation is considered as a first approximation of the Klein-Gordon equation

$$
u_{t t}-u_{x x}-a u+u^{1+2 \alpha}=0 \quad \text { in }(0, \pi) \times \mathbb{R} .
$$

Observe that (8) with $a=0$ and $\alpha=1$ is the meson equation of Schiff [4] (cf. also Jörgens [5]).

The physical motivation of (8) with $\alpha=1$ can be seen in Lourêdo et al. [6].

Problem (4) with $F(u)$ of the form (5) generalizes (8) when $a=0$. The existence of solutions of this problem is studied in Theorem 1 .

In Louredo et al., loc.cit., is analyzed the equation

$$
\begin{gathered}
u^{\prime \prime}-\mu(t) \Delta u+a\left(\int_{\Omega} u^{2} d x\right) u+b\left(\int_{\Omega} u^{\prime 2} d x\right) u^{\prime} \\
=0 \quad \text { in } \Omega \times(0, \infty)
\end{gathered}
$$

with nonlinear boundary condition. The $F(u)$ given in $(5)$ is different from the $a\left(\int_{\Omega} u^{2} d x\right)$ of this equation. The term $F(u)$ is related to the nonsmoothness of $f$.

\section{Main Results}

We use the notation $D\left(A^{\alpha}\right)^{\prime}=D\left(A^{-\alpha}\right), \alpha \in \mathbb{R}, \alpha \geq 0$. Identifying $H$ with $H^{\prime}$, we have

$$
D\left(A^{\alpha}\right) \hookrightarrow H \hookrightarrow D\left(A^{-\alpha}\right) .
$$

Here and in what follows the notation $X \hookrightarrow Y$ means that the space $X$ in dense in the space $Y$ and the embedding of $X$ in $Y$ are continuous. Note that $D\left(A^{-\alpha}\right)^{\prime}=D\left(A^{\alpha}\right)$. Also, if $\beta, \gamma \in \mathbb{R}$ with $\beta \geq \gamma$, we have

$$
D\left(A^{\beta}\right) \hookrightarrow D\left(A^{\gamma}\right) .
$$

Assume that

the embedding of $V$ in $H$ is compact.

First we analyze Problem (2) with $F(u)=\left|A^{-\theta / 2} u\right|^{2 p}$, that is, the problem

$$
\begin{gathered}
u^{\prime \prime}+\mu A u+\left|A^{-\theta / 2} u\right|^{2 p} u=f \quad \text { in }(0, T), \\
u(0)=u^{0}, \quad u^{\prime}(0)=u^{0}
\end{gathered}
$$

Theorem 1. Assume condition (13). Let $\theta$ and $p$ be real numbers with $p \geq 1$. Consider

$$
\begin{gathered}
u^{0} \in D\left(A^{(1-\theta) / 2}\right), \quad u^{1} \in D\left(A^{-\theta / 2}\right), \\
\mu \in W^{1,1}(0, T), \quad \mu(t) \geq \mu_{0}>0, \\
\forall t \in[0, T]\left(\mu_{0} \text { constant }\right), \\
f \in W^{1,1}\left(0, T ; D\left(A^{-(1+\theta) / 2}\right)\right) .
\end{gathered}
$$

Then there exists a function $u$ in the class

$$
\begin{gathered}
u \in L^{\infty}\left(0, T ; D\left(A^{(1-\theta) / 2}\right)\right), \\
u^{\prime} \in L^{\infty}\left(0, T ; D\left(A^{-\theta / 2}\right)\right), \\
u^{\prime \prime} \in L^{\infty}\left(0, T ; D\left(A^{-(1+\theta) / 2}\right)\right)
\end{gathered}
$$

such that $u$ is solution of the equation

$$
\begin{aligned}
u^{\prime \prime}+ & \mu A u+\left|A^{-\theta / 2} u\right|^{2 p} u \\
& =f \quad \text { in } L^{\infty}\left(0, T ; D\left(A^{-(1+\theta) / 2}\right)\right)
\end{aligned}
$$

and satisfies the initial conditions

$$
u(0)=u^{0}, \quad u^{\prime}(0)=u^{1} .
$$

Remark 2. When $\mu=1$ it is possible to obtain a solution $u$ of Problem (14) by using the Theory of Semigroups (cf. Pazy [7]).

To formulate the second problem, we introduce some notations. In fact, let us define

$$
Y=D\left(A^{(1-\theta) / 2}\right), \quad Z=D\left(A^{-\theta / 2}\right), \quad X=D\left(A^{\lambda}\right),
$$

where

$$
-\frac{\theta}{2} \leq \lambda<\frac{1-\theta}{2}, \quad \lambda \in \mathbb{R}
$$

By (12) we have

$$
Y \hookrightarrow X \hookrightarrow Z
$$

Consider Problem (4) with $F(u)=\|u\|_{C^{0}([0, T] ; X)}$ and $u_{0}=$ 0 , that is, the problem

$$
\begin{gathered}
u^{\prime \prime}+\mu A u+\|u\|_{C^{0}([0, T] ; X)} u=f \quad \text { in }(0, T), \\
u(0)=0, \quad u^{\prime}(0)=u^{1} .
\end{gathered}
$$

Theorem 3. Assume that $\theta, u^{1}$, and $f$ satisfy the hypotheses of Theorem 1 and $u^{0}=0$. Then there exists a function $u$ in the class (16) such that $u$ is solution of the problem

$$
\begin{aligned}
& u^{\prime \prime}+ \mu A u+\|u\|_{C^{0}([0, T] ; X)} u \\
&= f \quad \text { in } L^{\infty}\left(0, T ; D\left(A^{-(1+\theta) / 2}\right)\right), \\
& u(0)=0, \quad u^{\prime}(0)=u^{1} .
\end{aligned}
$$


Remark 4. Note that if $u$ belongs to class (16) then $u \in$ $C^{0}([0, T] ; X)$ (cf. Lions and Magenes [8]).

Corollary 5. Under the same hypotheses of Theorem 1, there exists a function $u$ in the class (16) such that $u$ is solution of the problem

$$
\begin{gathered}
u^{\prime \prime}+\mu A u+\|u\|_{L^{p}(0, T ; X)}^{p} u=f \quad \text { in } L^{\infty}\left(0, T ; D\left(A^{-(1+\theta) / 2}\right)\right), \\
u(0)=0, \quad u^{\prime}(0)=u^{1} .
\end{gathered}
$$

We analyze the uniqueness of solutions. Consider $\theta=0$ in Theorem 1 . Then the solution $u$ gives by this theorem when $\theta=0$ satisfies

$$
\begin{gathered}
u \in L^{\infty}\left(0, T ; D\left(A^{1 / 2}\right)\right), \\
u^{\prime} \in L^{\infty}(0, T ; H), \\
u^{\prime \prime} \in L^{\infty}\left(0, T ; D\left(A^{-1 / 2}\right)\right), \\
u^{\prime \prime}+\mu A u+|u|^{2 p} u=f \quad \text { in } L^{\infty}\left(0, T ; D\left(A^{-1 / 2}\right)\right), \\
u(0)=u^{0}, \quad u^{\prime}(0)=u^{1} .
\end{gathered}
$$

Theorem 6. Let $p \geq 1$ be a real number. Consider

$$
\begin{aligned}
& u^{0} \in D\left(A^{1 / 2}\right), \quad u^{1} \in H, \\
& \mu \text { satisfying }(15)_{2} \text { with } \mu^{\prime} \in L^{\infty}(0, T), \\
& f \in W^{1,1}\left(0, T ; D\left(A^{-1 / 2}\right)\right) .
\end{aligned}
$$

Then there exists a unique solution $u$ of Problem (26) in the class (25).

We do not know if there is uniqueness of solutions for Theorem 3, even when $\theta=0$.

In what follows we prove the above results.

\section{Proof of Theorem 1}

Before proving the theorem, we make some considerations on the operator $A^{\alpha}$. Recall hypothesis (13). By solving the spectral problem $((u, v))=\lambda(u, v)$, for all $v \in V$, we determine the eigenfunctions and eigenvalues, respectively, $\left(w_{\nu}\right)$ and $\left(\lambda_{\nu}\right)$ of the operator $A$, that is,

$$
\begin{gathered}
A w_{v}=\lambda_{\nu} w_{\nu}, \quad v=1,2, \ldots, \\
\lambda_{v} \longrightarrow \infty, \quad v \longrightarrow \infty .
\end{gathered}
$$

Note that $\left(w_{\gamma}\right)$ is a Hilbert basis of $H$ (cf. Brezis [9] and Komornik [10]).

Let be $\alpha \in \mathbb{R}, \alpha \geq 0$. Then the linear operator

$$
A^{\alpha}: D\left(A^{\alpha}\right) \longrightarrow H
$$

is continuous, bijective, and

$$
A^{\alpha} u=\sum_{\nu=1}^{\infty} \lambda_{\nu}^{\alpha}\left(u, w_{\nu}\right) w_{\nu}, \quad \forall u \in D\left(A^{\alpha}\right) .
$$

Also,

$$
\left(A^{\alpha}\right)^{-1}: H \longrightarrow D\left(A^{\alpha}\right)
$$

is given by

$$
\left(A^{\alpha}\right)^{-1} f=\sum_{\nu=1}^{\infty} \lambda_{\nu}^{-\alpha}\left(f, w_{\nu}\right) w_{\nu}, \quad \forall f \in H .
$$

These results can be found in Lions [1] and Medeiros and Milla Miranda [11].

Introduce the adjoint operator $\left(A^{\alpha}\right)^{*}$ of $A^{\alpha}$, that is,

$$
\begin{aligned}
\left(A^{\alpha}\right)^{*}: H & \longrightarrow D\left(A^{\alpha}\right)^{\prime} \\
\left\langle\left(A^{\alpha}\right)^{*} f, z\right\rangle_{X^{\prime} \times \mathscr{X}} & =\left(f, A^{\alpha} z\right), \quad \forall z \in \mathscr{X},
\end{aligned}
$$

where $\mathscr{X}=D\left(A^{\alpha}\right)$. Note that $H$ is identified with $H^{\prime}$. By the properties of $A^{\alpha}$, we have that

$$
\left(A^{\alpha}\right)^{*} \text { is linear, continuous and bijective. }
$$

Thus, the linear operator

$$
\left[\left(A^{\alpha}\right)^{*}\right]^{-1}: D\left(A^{\alpha}\right)^{\prime} \longrightarrow H
$$

is continuous and bijective.

Proposition 7. Let $f \in H$ and $g \in D\left(A^{\alpha}\right)^{\prime}$. Then one has the following.

(i) $\sum_{\nu=1}^{\infty} \lambda_{\nu}^{\alpha}\left(f, w_{\nu}\right)_{w_{\nu}}$ converges in $D\left(A^{\alpha}\right)^{\prime}$, and

$$
\left(A^{\alpha}\right)^{*} f=\sum_{\nu=1}^{\infty} \lambda_{\nu}^{\alpha}\left(f, w_{\nu}\right) w_{\nu}
$$

(ii) $\sum_{\nu=1}^{\infty} \lambda_{\nu}^{-\alpha}\left\langle g, w_{\nu}\right\rangle_{X^{\prime} \times X} w_{\nu}$ converges in $H$, and

$$
\left[\left(A^{\alpha}\right)^{*}\right]^{-1} g=\sum_{\nu=1}^{\infty} \lambda_{\nu}^{-\alpha}\left\langle g, w_{\nu}\right\rangle_{X^{\prime} \times \mathscr{X}} w_{\nu},
$$

where $\mathscr{X}=D\left(A^{\alpha}\right)$.

Proof. We prove (i). As $f \in H$, we have

$$
f=\sum_{\nu=1}^{\infty}\left(f, w_{v}\right) w_{v} .
$$

Consider $z \in \mathscr{X}$. Then noting that $\left\langle w_{\gamma}, w_{\mu}\right\rangle_{\mathscr{X}^{\prime} \times \mathscr{X}}=\left(w_{\gamma}, w_{\mu}\right)$, we obtain

$$
\begin{aligned}
& \left\langle\sum_{\nu=m}^{n} \lambda_{\nu}^{\alpha}\left(f, w_{\nu}\right) w_{\nu}, z\right\rangle_{X^{\prime} \times \mathscr{X}} \\
& \quad=\left\langle\sum_{\nu=m}^{n} \lambda_{\nu}^{\alpha}\left(f, w_{\nu}\right) w_{\nu}, \sum_{\mu=1}^{\infty}\left(z, w_{\mu}\right) w_{\mu}\right\rangle_{X^{\prime} \times X} \\
& =\sum_{\nu=m}^{n} \lambda_{\nu}^{\alpha}\left(f, w_{\nu}\right)\left(z, w_{\nu}\right) .
\end{aligned}
$$


On the other hand, by (30) we derive

$$
\begin{aligned}
\left(\sum_{\nu=m}^{n}\left(f, w_{\nu}\right) w_{\nu}, A^{\alpha} z\right) & =\left(\sum_{\nu=m}^{n}\left(f, w_{\nu}\right) w_{\nu}, \sum_{\mu=1}^{\infty} \lambda_{\mu}^{\alpha}\left(z, w_{\mu}\right) w_{\mu}\right) \\
& =\sum_{\nu=m}^{n} \lambda_{\nu}^{\alpha}\left(f, w_{\nu}\right)\left(z, w_{\nu}\right) .
\end{aligned}
$$

The last two expressions give

$$
\left\langle\sum_{\nu=m}^{n} \lambda_{\nu}^{\alpha}\left(f, w_{\nu}\right) w_{\nu}, z\right\rangle_{\mathscr{X}^{\prime} \times \mathscr{X}}=\left(\sum_{\nu=m}^{n}\left(f, w_{\nu}\right) w_{\nu}, A^{\alpha} z\right) .
$$

This and (38) provide that

$$
\sum_{\nu=m}^{n} \lambda_{\nu}^{\alpha}\left(f, w_{\nu}\right) w_{\nu} \longrightarrow 0 \quad \text { in } \mathscr{X}^{\prime} \text { as } m, n \longrightarrow \infty .
$$

So $(\mathrm{i})_{1}$ is proved. Taking the limit in (41) and observing (33) we obtain (36).

We prove (ii). We have that there exists a unique $f \in H$ such that

$$
\left(A^{\alpha}\right)^{*} f=g .
$$

By $(33)_{2}$ and $(30)_{1}$, we have

$$
\left\langle g, w_{\mu}\right\rangle_{X^{\prime} \times X}=\left(\sum_{\nu=1}^{\infty}\left(f, w_{\nu}\right) w_{\nu}, A^{\alpha} w_{\mu}\right)=\lambda_{\mu}^{\alpha}\left(f, w_{\mu}\right) .
$$

Then

$$
\sum_{\nu=m}^{n} \lambda_{\nu}^{-\alpha}\left\langle g, w_{\nu}\right\rangle_{X^{\prime} \times X^{\prime}} w_{\nu}=\sum_{\nu=m}^{n}\left(f, w_{\nu}\right) w_{\nu} .
$$

This implies that

$$
\sum_{\nu=m}^{n} \lambda_{\nu}^{-\alpha}\left\langle g, w_{\nu}\right\rangle_{X^{\prime} \times X^{\prime}} w_{\nu} \longrightarrow 0 \quad \text { in } H \text { as } m, n \longrightarrow \infty
$$

Thus (ii) $)_{2}$ is proved. By (43) and (45), we obtain

$$
\sum_{\nu=1}^{\infty} \lambda_{\nu}^{-\alpha}\left\langle g, w_{\nu}\right\rangle_{X^{\prime} \times X} w_{\nu}=\sum_{\nu=1}^{\infty}\left(f, w_{\nu}\right) w_{\nu}=f=\left[\left(A^{\alpha}\right)^{*}\right]^{-1} g .
$$

This concludes the proof of the proposition.

Motived by (37), we equip the space $D\left(A^{\alpha}\right)^{\prime}$ with the scalar product

$$
\begin{aligned}
(g, h)_{D\left(A^{\alpha}\right)^{\prime}} & \\
= & \left(\left[\left(A^{\alpha}\right)^{*}\right]^{-1} g,\left[\left(A^{\alpha}\right)^{*}\right]^{-1} h\right) \\
& =\left(\sum_{\nu=1}^{\infty} \lambda_{\nu}^{-\alpha}\left\langle g, w_{\nu}\right\rangle_{X^{\prime} \times \mathscr{X}} w_{\nu}, \sum_{\mu=1}^{\infty} \lambda_{\mu}^{-\alpha}\left\langle h, w_{\mu}\right\rangle_{X^{\prime} \times X^{\prime}} w_{\mu}\right),
\end{aligned}
$$

where $\mathscr{X}=D\left(A^{\alpha}\right)$. This scalar product on $\mathscr{X}^{\prime}$ yields a norm

$$
\|g\|_{D\left(A^{\alpha}\right)^{\prime}}=\left\|\left[\left(A^{\alpha}\right)^{*}\right]^{-1} g\right\|=\left|\sum_{\nu=1}^{\infty} \lambda_{\nu}^{-\alpha}\left\langle g, w_{\nu}\right\rangle_{X^{\prime} \times X^{\prime}} w_{\nu}\right|
$$

which is equivalent to the usual norm of $D\left(A^{\alpha}\right)^{\prime}$.

By similarity between expressions (30) and (36) and between (32) and (37), respectively, we introduce the notations

$$
\left(A^{\alpha}\right)^{*}=A^{\alpha}, \quad\left[\left(A^{\alpha}\right)^{*}\right]^{-1}=\left(A^{\alpha}\right)^{-1} .
$$

Also we use the notation

$$
\left(A^{\alpha}\right)^{-1}=A^{-\alpha}=D\left(A^{\alpha}\right)^{\prime}
$$

With these considerations and expressions (29) and (33), we obtain

$$
D\left(A^{\alpha}\right) \stackrel{A^{\alpha}}{\longrightarrow} H \stackrel{A^{\alpha}}{\longrightarrow} D\left(A^{-\alpha}\right)
$$

and by expressions (35) and (31),

$$
D\left(A^{-\alpha}\right) \stackrel{A^{-\alpha}}{\longrightarrow} H \stackrel{A^{-\alpha}}{\longrightarrow} D\left(A^{\alpha}\right) .
$$

Also by (37) and (48), (49), respectively, we find

$$
A^{-\alpha} g=\sum_{\nu=1}^{\infty} \lambda_{\nu}^{-\alpha}\left\langle g, w_{\nu}\right\rangle_{D\left(A^{-\alpha}\right) \times D\left(A^{\alpha}\right)} w_{\nu}, \quad \forall g \in D\left(A^{-\alpha}\right),
$$

$$
(g, h)_{D\left(A^{-\alpha}\right)}=\left(A^{-\alpha} g, A^{-\alpha} h\right), \quad\|g\|_{D\left(A^{-\alpha}\right)}=\left|A^{-\alpha} g\right| .
$$

Proposition 8. Consider $\beta, \gamma \in \mathbb{R}$. Then the linear operator

$$
A^{\gamma}: D\left(A^{\beta}\right) \longrightarrow D\left(A^{\gamma-\beta}\right)^{\prime}
$$

defined by

$$
\left\langle A^{\gamma} y, z\right\rangle_{D\left(A^{\gamma-\beta}\right)^{\prime} \times D\left(A^{\gamma-\beta}\right)}=\left(A^{\beta} y, A^{\gamma-\beta} z\right)
$$

is continuous.

Proof. We obtain

$$
\left|\left\langle A^{\gamma} y, z\right\rangle_{y^{\prime} \times y}\right| \leq\left|A^{\beta} y\right|\left|A^{\gamma-\beta} z\right|=\|y\|_{D\left(A^{\beta}\right)}\|z\| y,
$$

where $\mathcal{Y}=D\left(A^{\gamma-\beta}\right)$. Then

$$
\left\|A^{\gamma} y\right\|_{y^{\prime}} \leq\|y\|_{D\left(A^{\beta}\right)}, \quad \forall y \in D\left(A^{\beta}\right)
$$


Proof of Theorem 1. We use the Galerkin method (cf. Lions [12] and Vicente and Frota [13]). Thus consider an approximate solution $u_{m}$ of Problem (14); that is,

$$
\begin{gathered}
u_{m}(t)=\sum_{j=1}^{m} g_{j m}(t) w_{j} ; \\
\left(u_{m}^{\prime \prime}, w_{j}\right)+\mu\left(A u_{m}, w_{j}\right)+\left|A^{-\theta / 2} u_{m}\right|^{2 p}\left(u_{m}, w_{j}\right) \\
=\left\langle f, w_{j}\right\rangle_{D\left(A^{\gamma_{0}}\right)^{\prime} \times D\left(A^{\gamma_{0}}\right)^{\prime}} \quad j=1,2, \ldots, m ; \\
u_{m}(0)=u_{m}^{0}=\sum_{j=1}^{m}\left\langle u^{0}, w_{j}\right\rangle_{E^{\prime} \times E} w_{j} ; \\
u_{m}^{\prime}(0)=u_{m}^{1}=\sum_{j=1}^{m}\left\langle u^{1}, w_{j}\right\rangle_{G^{\prime} \times G} w_{j}
\end{gathered}
$$

where $E=D\left(A^{-(1-\theta) / 2}\right)$ and $G=D\left(A^{\theta / 2}\right)$.

Remark 9. Note that

$$
\begin{gathered}
u_{m}^{0} \longrightarrow u^{0} \quad \text { in } D\left(A^{(1-\theta) / 2}\right), \\
\left|A^{(1-\theta) / 2} u_{m}^{0}\right| \leq\left|A^{(1-\theta) / 2} u^{0}\right|, \\
u_{m}^{1} \longrightarrow u^{1} \quad \text { in } D\left(A^{-\theta / 2}\right), \\
\left|A^{-\theta / 2} u_{m}^{1}\right| \leq\left|A^{-\theta / 2} u^{1}\right| .
\end{gathered}
$$

Remark 10. Observe that if $\gamma \leq 0$ then

$$
\left\langle z, w_{j}\right\rangle_{D\left(A^{-\gamma}\right) \times D\left(A^{\gamma}\right)}=\left(z, w_{j}\right), \quad j=1,2, \ldots
$$

Multiply both sides of $(61)_{1}$ by $g_{j m}^{\prime}(t) \lambda_{j}^{-\theta}$ and add from $j=1$ up to $j=m$. We obtain

$$
\begin{aligned}
\left(u_{m}^{\prime \prime}, A^{-\theta} u_{m}^{\prime}\right)+\mu\left(A u_{m}, A^{-\theta} u_{m}^{\prime}\right) & \\
& +\left|A^{-\theta / 2} u_{m}\right|^{2 p}\left(u_{m}, A^{-\theta} u_{m}^{\prime}\right) \\
= & \left\langle f, A^{-\theta} u_{m}^{\prime}\right\rangle_{W^{\prime} \times W},
\end{aligned}
$$

where

$$
W=D\left(A^{\gamma_{0}}\right), \quad \gamma_{0}=\frac{1+\theta}{2} .
$$

Then,

$$
\begin{aligned}
\frac{1}{2} \frac{d}{d t}\left|A^{-\theta / 2} u_{m}^{\prime}\right|^{2}+\frac{1}{2} \frac{d}{d t}\left(\mu\left|A^{(1-\theta) / 2} u_{m}\right|^{2}\right) \\
+\frac{1}{2(p+1)} \frac{d}{d t}\left|A^{-\theta / 2} u_{m}\right|^{2(p+1)} \\
=\frac{1}{2} \mu^{\prime}\left|A^{(1-\theta) / 2} u_{m}\right|^{2}+\left\langle f, A^{-\theta} u_{m}^{\prime}\right\rangle_{W^{\prime} \times W} .
\end{aligned}
$$

By Proposition 8 with $\gamma=0$ and $\beta=-\gamma_{0}$ and noting that $\gamma_{0}-\theta=(1-\theta) / 2$, we obtain

$$
\left\langle f, A^{-\theta} w_{j}\right\rangle_{W^{\prime} \times W}=\left(A^{-\gamma_{0}} f, A^{\gamma_{0}-\theta} w_{j}\right)=\left(A^{-\gamma_{0}} f, A^{(1-\theta) / 2} w_{j}\right) .
$$

Substituting this equality into (66) and integrating on $[0, t]$, we obtain

$$
\begin{aligned}
\frac{1}{2}\left|A^{-\theta / 2} u_{m}^{\prime}(t)\right|^{2}+\frac{1}{2} \mu(t)\left|A^{(1-\theta) / 2} u_{m}(t)\right|^{2} \\
+\frac{1}{2(p+1)}\left|A^{-\theta / 2} u_{m}(t)\right|^{2(p+1)} \\
=\frac{1}{2} \int_{0}^{t} \mu^{\prime}(s)\left|A^{(1-\theta) / 2} u_{m}(s)\right|^{2} d s \\
+\int_{0}^{t}\left(A^{-\gamma_{0}} f(s), A^{(1-\theta) / 2} u_{m}^{\prime}(s)\right) d s \\
+\frac{1}{2}\left|A^{-\theta / 2} u_{m}^{1}\right|^{2}+\frac{1}{2} \mu(0)\left|A^{(1-\theta) / 2} u_{m}^{0}\right|^{2} \\
\quad+\frac{1}{2(p+1)}\left|A^{-\theta / 2} u_{m}^{0}\right|^{2(p+1)} .
\end{aligned}
$$

Note that

$$
\begin{aligned}
& \frac{1}{2} \int_{0}^{t} \mu^{\prime}(s)\left|A^{(1-\theta) / 2} u_{m}(s)\right|^{2} d s \\
& \quad \leq 2 \int_{0}^{t} \frac{\left|\mu^{\prime}(s)\right|}{\mu_{0}}\left[\frac{1}{4} \mu(s)\right]\left|A^{(1-\theta) / 2} u_{m}(s)\right|^{2} d s
\end{aligned}
$$

Also

$$
\begin{aligned}
\int_{0}^{t}\left(A^{-\gamma_{0}} f(s), A^{(1-\theta) / 2} u_{m}^{\prime}(s)\right) d s \\
=\left(A^{-\gamma_{0}} f(t), A^{(1-\theta) / 2} u_{m}(t)\right) \\
-\left(A^{-\gamma_{0}} f(0), A^{(1-\theta) / 2} u_{m}^{0}\right) \\
\quad-\int_{0}^{t}\left(A^{-\gamma_{0}} f^{\prime}(s), A^{(1-\theta) / 2} u_{m}(s)\right) d s,
\end{aligned}
$$

$$
\begin{aligned}
& \left|\left(A^{-\gamma_{0}} f(t), \mathrm{A}^{(1-\theta) / 2} u_{m}(t)\right)\right| \\
& \leq \frac{1}{\mu_{0}}\|f\|_{L^{\infty}\left(0, T ; D\left(A^{-\gamma_{0}}\right)\right)}^{2}+\frac{1}{4} \mu(t)\left|A^{(1-\theta) / 2} u_{m}(t)\right|^{2} \\
& \left|\int_{0}^{t}\left(A^{-\gamma_{0}} f^{\prime}(s), A^{(1-\theta) / 2} u_{m}(s)\right) d s\right| \\
& \leq \frac{1}{\mu_{0}} \int_{0}^{T}\left|A^{-\gamma_{0}} f^{\prime}(s)\right|^{2} d s \\
& \quad+\frac{1}{4} \int_{0}^{t}\left|A^{-\gamma_{0}} f^{\prime}(s)\right|\left[\mu(s)\left|A^{(1-\theta) / 2} u_{m}(s)\right|^{2} d s\right] .
\end{aligned}
$$


Substituting (69)-(71) into (68), we find

$$
\begin{aligned}
\frac{1}{2}\left|A^{-\theta / 2} u_{m}^{\prime}(t)\right|^{2}+\frac{1}{4} \mu(t)\left|A^{(1-\theta) / 2} u_{m}(t)\right|^{2} \\
\quad+\frac{1}{2(p+1)}\left|A^{-\theta / 2} u_{m}(t)\right|^{2(p+1)} \\
\leq 2 \int_{0}^{t} \frac{\left|\mu^{\prime}(s)\right|}{\mu_{0}}\left[\frac{1}{4} \mu(s)\left|A^{(1-\theta) / 2} u_{m}(s)\right|^{2} d s\right] \\
+M+\left|A^{-\gamma_{0}} f(0)\right|\left|A^{(1-\theta) / 2} u^{0}\right|+N \\
+\frac{1}{4} \int_{0}^{t}\left|A^{-\gamma_{0}} f^{\prime}(s)\right|\left[\mu(s)\left|A^{(1-\theta) / 2} u_{m}(s)\right|^{2} d s\right] \\
+E(0)
\end{aligned}
$$

where

$$
\begin{gathered}
M=\frac{1}{\mu_{0}}\|f\|_{L^{\infty}\left(0, T ; D\left(A^{-\gamma_{0}}\right)\right)}^{2}, \\
N=\frac{1}{\mu_{0}} \int_{0}^{T}\left|A^{-\theta / 2} f^{\prime}(s)\right| d s, \\
E(0)=\frac{1}{2}\left|A^{-\theta / 2} u^{1}\right|^{2}+\frac{1}{2} \mu(0)\left|A^{(1-\theta) / 2} u^{0}\right|^{2} \\
+\frac{1}{2(p+1)}\left|A^{-\theta / 2} u^{0}\right|^{2(p+1)} .
\end{gathered}
$$

Applying Gronwall inequality in (72), we deduce

$$
\begin{aligned}
\frac{1}{2}\left|A^{-\theta / 2} u_{m}^{\prime}(t)\right|^{2}+\frac{1}{4} \mu(t)\left|A^{(1-\theta) / 2} u_{m}(t)\right|^{2} \\
+\frac{1}{2(p+1)}\left|A^{-\theta / 2} u_{m}(t)\right|^{2(p+1)} \\
\leq\left(E(0)+M+\left|A^{-\gamma_{0}} f(0)\right|\left|A^{(1-\theta) / 2} u^{0}\right|+N\right) \\
\quad \times \exp ^{\int_{0}^{T}\left[\left(2 / \mu_{0}\right)\left|\mu^{\prime}(t)\right|+\left|A^{-\gamma_{0}} f^{\prime}(t)\right|\right] d t},
\end{aligned}
$$$$
\forall t \in[0, T] .
$$

With this inequality, we determine a subsequence of $\left(u_{m}\right)$, still denoted by $\left(u_{m}\right)$, and a function $u$ such that

$$
\begin{gathered}
u_{m} \longrightarrow u \text { weak star in } L^{\infty}\left(0, T ; D\left(A^{(1-\theta) / 2}\right)\right), \\
u_{m}^{\prime} \longrightarrow u^{\prime} \text { weak star in } L^{\infty}\left(0, T ; D\left(A^{-\theta / 2}\right)\right) .
\end{gathered}
$$

By (13) we have that

$$
D\left(A^{(1-\theta) / 2}\right) \text { is compactly embedding in } D\left(A^{-\theta / 2}\right)
$$

(cf. [1] and [11]). Then convergences (75) and Aubin-Lions Theorem (cf. [14]) imply

$$
u_{m} \rightarrow u \quad \text { in } L^{\infty}\left(0, T ; D\left(A^{-\theta / 2}\right)\right) \text {. }
$$

Therefore,

$$
\left|A^{-\theta / 2} u_{m}\right|^{2 p} \longrightarrow\left|A^{-\theta / 2} u\right|^{2 p} \quad \text { in } L^{\infty}(0, T) .
$$

This convergence and convergence $(75)_{1}$ provide

$$
\begin{aligned}
& \left|A^{-\theta / 2} u_{m}\right|^{2 p} u_{m} \longrightarrow\left|A^{-\theta / 2} u\right|^{2 p} u \\
& \text { weak star in } L^{\infty}\left(0, T ; D\left(A^{(1-\theta) / 2}\right)\right)
\end{aligned}
$$

which implies

$$
\begin{aligned}
& \left|A^{-\theta / 2} u_{m}\right|^{2 p} u_{m} \longrightarrow\left|A^{-\theta / 2} u\right|^{2 p} u \\
& \text { weak star in } L^{\infty}\left(0, T ; D\left(A^{-\gamma_{0}}\right)\right)
\end{aligned}
$$

since $(1-\theta) / 2>-\gamma_{0}, \gamma_{0}$ defined in (65).

In order to obtain an estimate for $\left(u_{m}^{\prime \prime}\right)$, we apply the method of projections to the approximate equation (61) (cf. Lions [12]). Thus, we consider the orthogonal projection

$$
P_{m}: H \longrightarrow V_{m} \subset H, \quad P_{m} z=\sum_{j=1}^{m}\left(z, w_{j}\right) w_{j},
$$

where $V_{m}$ is the subspace generated by $w_{1}, w_{2}, \ldots, w_{m}$.

By similar arguments employed to obtain (67) and by (54) or (30), we find

$$
\left\langle f, w_{j}\right\rangle_{D\left(A^{\left.-\gamma_{0}\right) \times D\left(A^{\gamma_{0}}\right)}\right.}=\left(A^{-\gamma_{0}} f, A^{\gamma_{0}} w_{j}\right)=\lambda_{j}^{\gamma_{0}}\left(A^{-\gamma_{0}} f, w_{j}\right) .
$$

Multiply both members of $(61)_{1}$ by $\lambda_{j}^{-\gamma_{0}} w_{j}$ and add from $j=1$ up to $j=m$. Then, applying to this result, expression (82), affirmation (54), or (30) and noting that $A^{-\gamma_{0}} u_{m}^{\prime \prime}, A^{-\gamma_{0}}\left(A u_{m}\right),\left|A^{-\theta / 2} u_{m}\right|^{2 p} A^{-\gamma_{0}} u_{m}$ belong to $V_{m}$, we obtain

$$
\begin{gathered}
A^{-\gamma_{0}} u_{m}^{\prime \prime}+\mu A^{-\gamma_{0}}\left(A u_{m}\right)+\left|A^{-\theta / 2} u_{m}\right|^{2 p} A^{-\gamma_{0}} u_{m} \\
=P_{m}\left(A^{-\gamma_{0}} f\right)
\end{gathered}
$$

which gives

$$
\begin{aligned}
\left|A^{-\gamma_{0}} u_{m}^{\prime \prime}\right| \leq & \mu\left|A^{(1-\theta) / 2} u_{m}\right| \\
& +C\left|A^{-\theta / 2} u_{m}\right|^{2 p}\left|A^{(1-\theta) / 2} u_{m}\right|+\left|A^{-\gamma_{0}} f\right| .
\end{aligned}
$$

Then estimates (74) and (80) provide

$$
\left(u_{m}^{\prime \prime}\right) \text { is bounded in } L^{\infty}\left(0, T ; D\left(A^{-\gamma_{0}}\right)\right) .
$$

Thus, there exists a subsequence of $\left(u_{m}^{\prime \prime}\right)$, still denoted by $\left(u_{m}^{\prime \prime}\right)$, such that

$$
u_{m}^{\prime \prime} \longrightarrow u^{\prime \prime} \text { weak star in } L^{\infty}\left(0, T ; D\left(A^{-\gamma_{0}}\right)\right) .
$$

Expressions (75) and (86) tell us that $u$ belongs to class (16). Convergences $(75)_{1},(80)$, and (86) allow us to pass to limit in (83) and to obtain

$$
A^{-\gamma_{0}} u^{\prime \prime}+A^{-\gamma_{0}}(\mu A u)+\left|A^{-\theta / 2} u\right|^{2 p} A^{-\gamma_{0}} u=A^{-\gamma_{0}} f
$$

which provides (17). Initial conditions (18) follow from convergences (75) and (86). 


\section{Proof of Theorem 3}

The idea is to apply a fixed point argument to the problem

$$
\begin{gathered}
u_{k}^{\prime \prime}+\mu A u_{k}+k u_{k}=f \quad \text { in }(0, T), \\
u_{k}(0)=0, \quad u_{k}^{\prime}(0)=u^{1},
\end{gathered}
$$

where $k \in \mathbb{R}, k \geq 0$.

We solve (88). Consider an approximate solution $u_{k m}$ of (88) given by

$$
\begin{gathered}
u_{k m}(t)=\sum_{j=1}^{m} g_{j k m}(t) w_{j}, \\
\left(u_{k m}^{\prime \prime}, w_{j}\right)+\mu\left(A u_{k m}, w_{j}\right)+k\left(u_{k m}, w_{j}\right) \\
=\left\langle f, w_{j}\right\rangle_{D\left(A^{\gamma_{0}}\right)^{\prime} \times D\left(A^{\gamma_{0}}\right)^{\prime}} \quad j=1,2, \ldots, m ; \\
u_{k m}(0)=0, \quad u_{k m}^{\prime}(0)=u_{m}^{1} .
\end{gathered}
$$

By similar arguments used to obtain (74), we derive

$$
\begin{gathered}
\frac{1}{2}\left|A^{-\theta / 2} u_{k m}^{\prime}(t)\right|^{2}+\frac{1}{4} \mu(t)\left|A^{(1-\theta) / 2} u_{k m}(t)\right|^{2} \\
+\frac{k}{2}\left|A^{-\theta / 2} u_{k m}(t)\right|^{2} \\
\leq L \exp ^{\int_{0}^{T} a(t) d t}=R,
\end{gathered}
$$$$
\forall t \in[0, \mathrm{~T}], \forall k \geq 0, \forall m,
$$

where

$$
\begin{gathered}
L=\frac{1}{2}\left|A^{-\theta / 2} u^{1}\right|^{2}+M+N \quad(M \text { and } N \text { defined in (73)), } \\
a(t)=\frac{2}{\mu_{0}}\left|\mu^{\prime}(t)\right|+\left|A^{-\gamma_{0}} f^{\prime}(t)\right| .
\end{gathered}
$$

The preceding inequality gives

$$
\begin{array}{r}
\left(u_{k m}\right) \text { is bounded in } L^{\infty}\left(0, T ; D\left(A^{(1-\theta) / 2}\right)\right), \\
\forall m, \forall k \geq 0 ; \\
\left(u_{k m}^{\prime}\right) \text { is bounded in } L^{\infty}\left(0, T ; D\left(A^{-\theta / 2}\right)\right),
\end{array}
$$

$$
\forall m, \forall k \geq 0 .
$$

By the projection method, we obtain, as in (83),

$$
A^{-\gamma_{0}} u_{k m}^{\prime \prime}+\mu A^{-\gamma_{0}}\left(A u_{k m}\right)+k A^{-\gamma_{0}} u_{k m}=P_{m}\left(A^{-\gamma_{0}} f\right) \text {. }
$$

This and estimate (91) provide

$$
\left(u_{k m}^{\prime \prime}\right) \text { is bounded in } L^{\infty}\left(0, T ; D\left(A^{-\gamma_{0}}\right)\right), \quad \forall m .
$$

Estimates (93) and (95) allow us to find a subsequence of $\left(u_{k m}\right)$, still denoted by $\left(u_{k m}\right)$, and a function $u_{k}$ such that, by passing to limit in (94), we obtain

$$
\begin{array}{r}
A^{-\gamma_{0}} u_{k}^{\prime \prime}+A^{-\gamma_{0}} \mu\left(A u_{k}\right)+k A^{-\gamma_{0}} u_{k} \\
=A^{-\gamma_{0}} f \quad \text { in } L^{\infty}(0, T ; H) .
\end{array}
$$

This, initial conditions $(90)_{2}$, and estimates (95) imply

$$
\begin{gathered}
u_{k}^{\prime \prime}+\mu A u_{k}+k u_{k}=f \quad \text { in } L^{\infty}\left(0, T ; D\left(A^{-\gamma_{0}}\right)\right) ; \\
u_{k}(0)=0, \quad u_{k}^{\prime}(0)=u^{1} .
\end{gathered}
$$

By taking the lim inf in both side of (91), we obtain

$$
\left\|u_{k}^{\prime}\right\|_{L^{2}(0, T ; Z)}^{2}+\left\|u_{k}\right\|_{L^{2}(0, T ; Y)}^{2} \leq P, \quad \forall k \geq 0 .
$$

As $Y \hookrightarrow X \hookrightarrow Z$ and the embedding $Y$ in $X$ are compact $(\lambda<(1-\theta) / 2)$, it follows from of Aubin-Lions Theorem (see Simon [14]) that

$$
\left\|u_{k}\right\|_{C^{0}([0, T] ; X)} \leq P_{1}, \quad \forall k \geq 0 .
$$

Define the map

$$
\psi:[0, \infty) \longrightarrow \mathbb{R}, \quad \psi(k)=\left\|u_{k}\right\|_{C^{0}([0, T] ; X)},
$$

where $u_{k}$ is the solution of Problem (97). We will prove that $\psi$ has a fixed point. Consider only the case $f \neq 0$. The case $f=0$ is outside of our attention. We will prove the following results.

(I) One has $\psi(0)>0$.

In fact if $\psi(0)=0$, we have that $u_{0}=0$ is a solution of (97) with $k=0$, but this a contradiction since $f \neq 0$.

(II) One has $\psi$ is continuous on $[0, \infty)$.

Let $k_{0}>0$. Consider $k>0$. By $(94)$ and $(90)_{2}$ we obtain

$$
\begin{gathered}
u_{k m}^{\prime \prime}+\mu A u_{k m}+k u_{k m}=A^{\gamma_{0}} P_{m}\left(A^{-\gamma_{0}} f\right) ; \\
u_{k m}(0)=0, \quad u_{k m}^{\prime}(0)=u_{m}^{1}, \\
u_{k_{0} m}^{\prime \prime}+\mu A u_{k_{0} m}+k_{0} u_{k_{0} m}=A^{\gamma_{0}} P_{m}\left(A^{-\gamma_{0}} f\right), \\
u_{k_{0} m}(0)=0, \quad u_{k_{0} m}^{\prime}(0)=u_{m}^{1} .
\end{gathered}
$$

Use the notation $z_{m}=u_{k m}-u_{k_{0} m}$. Then the preceding problems give

$$
\begin{gathered}
z_{m}^{\prime \prime}+\mu A z_{m}+k_{0} z_{m}=-\left(k-k_{0}\right) u_{k m} ; \\
z_{m}(0)=0, \quad z_{m}^{\prime}(0)=0 .
\end{gathered}
$$

Taking the scalar product of $H$ of both sides of this equation with $A^{-\theta} z_{m}^{\prime}$, we find

$$
\begin{aligned}
\frac{1}{2} \frac{d}{d t}\left|A^{-\theta / 2} z_{m}^{\prime}\right|^{2} & +\frac{1}{2} \frac{d}{d t}\left[\mu\left|A^{(1-\theta) / 2} z_{m}\right|^{2}\right] \\
& +\frac{k_{0}}{2} \frac{d}{d t}\left|A^{-\theta / 2} z_{m}\right|^{2} \\
= & \frac{1}{2} \mu^{\prime}\left|A^{(1-\theta) / 2} z_{m}\right|^{2}-\left(k-k_{0}\right)\left(u_{k m}, A^{-\theta} z_{m}^{\prime}\right) .
\end{aligned}
$$

We have

$$
\left(u_{k m}, A^{-\theta} z_{m}^{\prime}\right)=\left(A^{-\gamma_{0}} u_{k m}, A^{(1-\theta) / 2} z_{m}^{\prime}\right) .
$$


Integrating on $[0, t]$ both sides of the last two expressions, we derive

$$
\begin{aligned}
\frac{1}{2}\left|A^{-\theta / 2} z_{m}^{\prime}(t)\right|^{2}+\frac{1}{2} \mu(t)\left|A^{(1-\theta) / 2} z_{m}(t)\right|^{2} \\
+\frac{k_{0}}{2}\left|A^{-\theta / 2} z_{m}(t)\right|^{2} \\
=\frac{1}{2} \int_{0}^{t} \mu^{\prime}(s)\left|A^{(1-\theta) / 2} z_{m}(s)\right|^{2} d s \\
-\left(k-k_{0}\right) \int_{0}^{t}\left(A^{-\gamma_{0}} u_{k m}(s), A^{(1-\theta) / 2} z_{m}^{\prime}(s)\right) d s .
\end{aligned}
$$

We obtain

$$
\begin{aligned}
\int_{0}^{t}\left(A^{-\gamma_{0}} u_{k m}(s), A^{(1-\theta) / 2} z_{m}^{\prime}(s)\right) d s \\
=\left(A^{-\gamma_{0}} u_{k m}(t), A^{(1-\theta) / 2} z_{m}(t)\right) \\
\quad-\int_{0}^{t}\left(A^{-\gamma_{0}} u_{k m}^{\prime}(s), A^{(1-\theta) / 2} z_{m}(s)\right) d s
\end{aligned}
$$

As $(1-\theta) / 2>-\gamma_{0}$, we have

$$
\begin{aligned}
& \left|\left(A^{-\gamma_{0}} u_{k m}(t), A^{(1-\theta) / 2} z_{m}(t)\right)\right| \\
& \quad \leq C\left|A^{(1-\theta) / 2} u_{k m}(t)\right|\left|A^{(1-\theta) / 2} z_{m}(t)\right| \\
& \quad \leq \frac{C^{2}}{2 \mu_{0}}\left|A^{(1-\theta) / 2} u_{k m}(t)\right|+\frac{1}{2} \mu(t)\left|A^{(1-\theta) / 2} z_{m}(t)\right|^{2} .
\end{aligned}
$$

Also,

$$
\begin{gathered}
\left(A^{-\gamma_{0}} u_{k m}^{\prime}, A^{(1-\theta) / 2} z_{m}\right)=\left(A^{-\theta / 2} u_{k m}^{\prime}, A^{-\theta / 2} z_{m}\right), \\
\left|\int_{0}^{t}\left(A^{-\gamma_{0}} u_{k m}^{\prime}(s), A^{(1-\theta) / 2} z_{m}(s)\right) d s\right| \\
\leq \int_{0}^{t}\left|A^{-\theta / 2} u_{k m}^{\prime}(s)\right|\left|A^{-\theta / 2} z_{m}(s)\right| d s \\
\leq \int_{0}^{t} C\left|A^{-\theta / 2} u_{k m}^{\prime}(s)\right|\left|A^{(1-\theta) / 2} z_{m}(t)\right| d s \\
\leq \frac{C^{2}}{2 \mu_{0}} \int_{0}^{T}\left|A^{-\theta / 2} u_{k m}^{\prime}(t)\right|^{2} d t \\
\quad+\frac{1}{2} \int_{0}^{t} \mu(s)\left|A^{(1-\theta) / 2} z_{m}(s)\right|^{2} d s .
\end{gathered}
$$
find

Taking into account estimate (91) in (107) and (109), we

$$
\begin{aligned}
& \left|\left(A^{-\gamma_{0}} u_{k m}(t), A^{(1-\theta) / 2} z_{m}(t)\right)\right| \\
& \quad \leq \frac{2 C^{2} R}{\mu_{0}^{2}}+\frac{1}{2} \mu(t)\left|A^{(1-\theta) / 2} z_{m}(t)\right|^{2}, \\
& \left|\int_{0}^{t}\left(A^{-\gamma_{0}} u_{k m}^{\prime}(s), A^{(1-\theta) / 2} z_{m}(s)\right) d s\right| \\
& \quad \leq \frac{C^{2} R T}{\mu_{0}}+\frac{1}{2} \int_{0}^{t} \mu(s)\left|A^{(1-\theta) / 2} z_{m}(s)\right|^{2} d s .
\end{aligned}
$$

Substituting the last two inequalities into (106), we obtain

$$
\begin{aligned}
\left|k-k_{0}\right| & \int_{0}^{t}\left|\left(A^{-\gamma_{0}} u_{k m}(s), A^{(1-\theta) / 2} z_{m}^{\prime}(s)\right)\right| d s \\
\leq & \frac{2 C^{2} R}{\mu_{0}^{2}}\left|k-k_{0}\right| \\
& +\frac{1}{2}\left|k-k_{0}\right| \mu(t)\left|A^{(1-\theta) / 2} z_{m}(t)\right|^{2}+\frac{C^{2} R T}{\mu_{0}}\left|k-k_{0}\right| \\
& +\frac{1}{2}\left|k-k_{0}\right| \int_{0}^{t} \mu(s)\left|A^{(1-\theta) / 2} z_{m}(s)\right|^{2} d s .
\end{aligned}
$$

Combining this inequality with (105), we derive

$$
\begin{aligned}
\frac{1}{2}\left|A^{-\theta / 2} z_{m}^{\prime}(t)\right|^{2}+\frac{\left(1-\left|k-k_{0}\right|\right)}{2} \mu(t)\left|A^{(1-\theta) / 2} z_{m}(t)\right|^{2} \\
+\frac{k_{0}}{2}\left|A^{-\theta / 2} z_{m}(t)\right|^{2} \\
\leq \frac{1}{2 \mu_{0}} \int_{0}^{t}\left|\mu^{\prime}(s)\right|\left[\mu(s)\left|A^{(1-\theta) / 2} z_{m}(s)\right|^{2}\right] d s \\
+\left[\frac{2 C^{2} R}{\mu_{0}^{2}}+\frac{C^{2}}{\mu_{0}} R T\right]\left|k-k_{0}\right| \\
+\frac{1}{2}\left|k-k_{0}\right| \int_{0}^{t} \mu(s)\left|A^{(1-\theta) / 2} z_{m}(s)\right|^{2} d s .
\end{aligned}
$$

Considering $\left|k-k_{0}\right|<1 / 2$ and using the Gronwall inequality, this expression gives

$$
\begin{array}{r}
\frac{1}{2}\left|A^{-\theta / 2} z_{m}^{\prime}(t)\right|^{2}+\frac{1}{4} \mu_{0}\left|A^{(1-\theta) / 2} z_{m}(t)\right|^{2} \\
+\frac{k_{0}}{2}\left|A^{-\theta / 2} z_{m}(t)\right|^{2} \leq R_{1}\left|k-k_{0}\right|, \\
\forall t \in[0, T],
\end{array}
$$


where the constant $R_{1}>0$ is independent of $m$ and $k$. Taking the lim inf in both sides of this inequality, we find

$$
\begin{aligned}
\frac{1}{2} \mid A^{-\theta / 2} & \left.\left(u_{k}^{\prime}(t)-u_{k_{0}}^{\prime}(t)\right)\right|^{2} \\
& +\frac{\mu_{0}}{4}\left|A^{(1-\theta) / 2}\left(u_{k}(t)-u_{k_{0}}(t)\right)\right|^{2} \\
\leq & R_{1}\left|k-k_{0}\right|, \quad \forall t \in[0, T] .
\end{aligned}
$$

By Simon [14] and noting that the embedding of $Y$ in $X$ is compact, we derive

$$
u_{k} \longrightarrow u_{k_{0}} \quad \text { in } C^{0}([0, T] ; X), \quad k \longrightarrow k_{0}
$$

Thus.

$$
\left\|u_{k}\right\|_{C^{0}([0, T] ; X)} \longrightarrow\left\|u_{k_{0}}\right\|_{C^{0}([0, T] ; X)}, \quad k \longrightarrow k_{0}
$$

which proves the continuity of $\psi$ at $k_{0}>0$. In similar way we prove the continuity of $\psi$ at $k_{0}=0$.

(III) One has $\psi(k) \rightarrow 0$ as $k \rightarrow \infty$.

Let $\left(k_{l}\right)$ be a sequence of positive numbers with $k_{l} \rightarrow \infty$. It follows from (98) and the compactness of the embedding of $Y$ in $X$ that there exists a subsequence of $\left(k_{l}\right)$, still denoted by $\left(k_{l}\right)$, and a function $\chi$ such that

$$
u_{k_{l}} \longrightarrow \chi \quad \text { in } C^{0}([0, T] ; X)
$$

This implies

$$
u_{k_{l}} \longrightarrow \chi \quad \text { in } L^{2}(0, T ; Z) .
$$

By estimate (91), we obtain

$$
\frac{k}{2}\left\|u_{k}\right\|_{L^{2}(0, T ; Z)}^{2} \leq R, \quad \forall k \geq 0 .
$$

Then

$$
u_{k_{l}} \longrightarrow 0 \quad \text { in } L^{2}(0, T ; Z) .
$$

Convergences (118) and (120) provide

$$
\chi=0 .
$$

Thus by (117) we find

$$
u_{k_{l}} \longrightarrow 0 \quad \text { in } C^{0}([0, T] ; X) .
$$

As the sequence $\left(k_{l}\right)$ was arbitrary and the limit of $\left(u_{k_{l}}\right)$ is always the same, we conclude that

$$
u_{k} \longrightarrow 0 \quad \text { in } C^{0}([0, T] ; X), \quad k \longrightarrow \infty .
$$

Thus

$$
\left\|u_{k}\right\|_{C^{0}([0, T] ; X)} \longrightarrow 0, \quad k \longrightarrow \infty
$$

which proves part (III).
By (I)-(III), we deduce that there exists $k>0, k \in \mathbb{R}$ such that

$$
\psi(k)=k .
$$

Considering this $k$ in (97), we obtain a solution $u$ of (22) that satisfies all conditions of the theorem.

The proof of Corollary 5 follows by defining the map

$$
\psi:[0, \infty) \longrightarrow \mathbb{R}, \quad \psi(k)=\left\|u_{k}\right\|_{L^{p}(0, T ; X)}^{p},
$$

where $u_{k}$ is the solution of the problem

$$
\begin{aligned}
u_{k}^{\prime \prime}+\mu A u_{k}+k u_{k} & =f \quad \text { in } L^{\infty}\left(0, T ; D\left(A^{-(1+\theta) / 2}\right)\right) ; \\
u(0) & =0, \quad u^{\prime}(0)=u^{1},
\end{aligned}
$$

and applying similar arguments to those used in the proof of Theorem 3.

\section{Proof of Theorem 6}

Let $u$ and $z$ be solutions of Problem (26) with $u$ and $z$ in class (25). Consider $w=u-z$. Then by (26) we have

$$
\begin{gathered}
w^{\prime \prime}+\mu A w+|u|^{2 p} w+\left(|u|^{2 p}-|z|^{2 p}\right) z \\
=0 \quad \text { in } L^{\infty}\left(0, T ; D\left(A^{-1 / 2}\right)\right), \\
w(0)=0, \quad w^{\prime}(0)=0 .
\end{gathered}
$$

Fix $0<s \leq T$. Consider $0 \leq t \leq s$. Introduce the function

$$
y(t)=\int_{s}^{t} w(\tau) d \tau
$$

We have

$$
\begin{gathered}
y \in C^{0}\left([0, s] ; D\left(A^{1 / 2}\right)\right) ; \\
y(s)=0, \quad y^{\prime}(t)=w(t) \quad \text { a.e. } t \in(0, s) ; \\
A^{1 / 2} y(t)=\int_{s}^{t} A^{1 / 2} w(\tau) d \tau, \quad A^{1 / 2} y^{\prime}(t)=A^{1 / 2} w(t) ; \\
y(t)=-\int_{0}^{s} w(\tau) d \tau+\int_{0}^{t} w(\tau) d \tau .
\end{gathered}
$$

Apply the operator given by the first member of $(128)_{1}$ to $y(t)$. We obtain

$$
\begin{aligned}
\left\langle w^{\prime \prime}(t), y(t)\right\rangle_{E^{\prime} \times E}+\mu(t)\langle A w(t), y(t)\rangle_{E^{\prime} \times E} \\
+|u(t)|^{2 p}(w(t), y(t)) \\
\quad+\left(|u(t)|^{2 p}-|z(t)|^{2 p}\right)(z(t), y(t)) \\
=0, \quad \text { a.e. } t \in(0, s),
\end{aligned}
$$


where $E=D\left(A^{1 / 2}\right)$. By $(130)_{2}$, we find

$$
\left\langle w^{\prime \prime}(t), y(t)\right\rangle_{E^{\prime} \times E}=\frac{d}{d t}\left\langle w^{\prime}(t), w(t)\right\rangle-\frac{1}{2} \frac{d}{d t}|w(t)|^{2} .
$$

Also by $(130)_{3}$,

$$
\begin{aligned}
\mu(t)\langle A w(t), y(t)\rangle_{E^{\prime} \times E}= & \frac{1}{2} \frac{d}{d t}\left[\mu(t)\left|A^{1 / 2} y(t)\right|^{2}\right] \\
& -\frac{1}{2} \mu^{\prime}(t)\left|A^{1 / 2} y(t)\right|^{2} .
\end{aligned}
$$

Integrate (131) on $[0, s]$ and use (128) $)_{1},(130),(132)$, and (133). We deduce

$$
\begin{aligned}
\frac{1}{2}|w(s)|^{2} & +\frac{1}{2} \mu(0)\left|\int_{0}^{s} A^{1 / 2} w(t) d t\right|^{2} \\
= & -\frac{1}{2} \int_{0}^{s} \mu^{\prime}(t)\left|A^{1 / 2} y(t)\right|^{2} d t \\
& +\int_{0}^{s}|u(t)|^{2 p}(w(t), y(t)) d t \\
& +\int_{0}^{s}\left[|u(t)|^{2 p}-|z(t)|^{2 p}\right](z(t), y(t)) d t .
\end{aligned}
$$

We modify each term of the second member of (134). We have

$$
A^{1 / 2} y(t)=\int_{0}^{t} A^{1 / 2} w(\tau) d \tau-\int_{0}^{s} A^{1 / 2} w(\tau) d \tau .
$$

Then

$$
\begin{aligned}
& \int_{0}^{s}\left|\mu^{\prime}(t)\right|\left|A^{1 / 2} y(t)\right|^{2} d t \\
& \leq 2 \int_{0}^{s}\left|\mu^{\prime}(t)\right|\left|\int_{0}^{t} A^{1 / 2} w(\tau) d \tau\right|^{2} d t \\
& \quad+2 \int_{0}^{s}\left|\mu^{\prime}(t)\right|\left|\int_{0}^{s} A^{1 / 2} w(\tau) d \tau\right|^{2} d t \\
& \leq 2 \int_{0}^{s} \frac{\left|\mu^{\prime}(t)\right|}{\mu_{0}} \mu(0)\left|\int_{0}^{t} A^{1 / 2} w(\tau) d \tau\right|^{2} d t \\
& \quad+2\left|\int_{0}^{s} A^{1 / 2} w(\tau) d \tau\right|^{2} \int_{0}^{s}\left|\mu^{\prime}(t)\right| d t .
\end{aligned}
$$

First, we assume that $\mu^{\prime} \neq 0$. In this case, we choose $s_{0}=$ $\min \left\{\mu(0) / 8\left\|\mu^{\prime}\right\|_{L^{\infty}(0, T)}, 1, T\right\}>0$. We have

$$
\begin{array}{r}
2\left|\int_{0}^{s} A^{1 / 2} w(\tau) d \tau\right|^{2} \int_{0}^{s}\left|\mu^{\prime}(t)\right| d t \\
\leq \frac{1}{4} \mu(0)\left|\int_{0}^{s} A^{1 / 2} w(\tau) d \tau\right|^{2}, \\
0 \leq s \leq s_{0} .
\end{array}
$$

Combining this last inequality with (136), we find

$$
\begin{aligned}
& \int_{0}^{s}\left|\mu^{\prime}(t) \| A^{1 / 2} y(t)\right|^{2} d t \\
& \leq 2 \int_{0}^{s} \frac{\left|\mu^{\prime}(t)\right|}{\mu_{0}}\left[\mu(0)\left|\int_{0}^{t} A^{1 / 2} w(\tau) d \tau\right|^{2}\right] d t \\
& \quad+\frac{1}{4} \mu(0)\left|\int_{0}^{s} A^{1 / 2} w(\tau) d \tau\right|^{2}, \quad 0 \leq s \leq s_{0} .
\end{aligned}
$$

We introduce the notations

$$
\|u\|_{L^{\infty}(0, T ; H)} \leq S_{1}, \quad\|z\|_{L^{\infty}(0, T ; H)} \leq S_{2} .
$$

(Note that $w \in C^{0}([0, T] ; H)$ since $w$ belongs to class (25)). We have

$$
\begin{aligned}
& \left.\left|\int_{0}^{s}\right| u(t)\right|^{2 p}(w(t), y(t)) d t \mid \\
& \quad \leq \frac{1}{2} S_{1}^{2 p} \int_{0}^{s}|w(t)|^{2} d t+\frac{1}{2} S_{1}^{2 p} \int_{0}^{s}|y(t)|^{2} d t .
\end{aligned}
$$

By $(130)_{4}$, we obtain

$$
\begin{aligned}
|y(t)|^{2} & \leq 2\left[\int_{0}^{s}|w(\tau)| d \tau\right]^{2}+2\left[\int_{0}^{t}|w(\tau)| d \tau\right]^{2} \\
& \leq 4 s \int_{0}^{s}|w(\tau)|^{2} d \tau .
\end{aligned}
$$

Therefore

$$
\int_{0}^{s}|y(t)|^{2} d t \leq 4 s^{2} \int_{0}^{s}|w(t)|^{2} d t
$$

Combining (140) and (142), we deduce

$$
\begin{aligned}
& \left.\left|\int_{0}^{s}\right| u(t)\right|^{2 p}(w(t), y(t)) d t \mid \\
& \quad \leq S_{1}^{2 p} \int_{0}^{s} \frac{1}{2}|w(t)|^{2} d t+4 S_{1}^{2 p} s^{2} \int_{0}^{s} \frac{1}{2}|w(t)|^{2} d t .
\end{aligned}
$$

The preceding inequality with $0 \leq s \leq s_{0}$ gives

$$
\left.\left.\left|\int_{0}^{s}\right| u(t)\right|^{2 p}(w(t), y(t)) d t\left|\leq 5 S_{1}^{2 p} \int_{0}^{s} \frac{1}{2}\right| w(t)\right|^{2} d t .
$$

On the other hand,

$$
\begin{gathered}
|u(t)|^{2 p}-|z(t)|^{2 p}=\left(|u(t)|^{p}+|z(t)|^{p}\right)\left(|u(t)|^{p}-|z(t)|^{p}\right), \\
\left.|| u(t)\right|^{p}-|z(t)|^{p}\left|\leq p\left(S_{1}+S_{2}\right)^{p-1}\right| w(t) \mid .
\end{gathered}
$$

Hence,

$$
\left.|| u(t)\right|^{2 p}-|z(t)|^{2 p}|\leq N| w(t) \mid,
$$

where

$$
N=p\left(S_{1}^{p}+S_{2}^{p}\right)\left(S_{1}+S_{2}\right)^{p-1} .
$$


Thus estimate (146) gives

$$
\begin{aligned}
\left|\int_{0}^{s}\left(|u(t)|^{2 p}-|z(t)|^{2 p}\right)(z(t), y(t)) d t\right| \\
\quad \leq N S_{2} \int_{0}^{s}|w(t)||y(t)| d t \\
\quad \leq N S_{2} \int_{0}^{s} \frac{1}{2}|w(t)|^{2} d t+\frac{1}{2} N S_{2} \int_{0}^{s}|y(t)|^{2} d t .
\end{aligned}
$$

This expressions, (142), and $0 \leq s \leq s_{0}$ provide

$$
\begin{gathered}
\left|\int_{0}^{s}\left(|u(t)|^{2 p}-|z(t)|^{2 p}\right)(z(t), y(t)) d t\right| \\
\leq 5 N S_{2} \int_{0}^{s} \frac{1}{2}|w(t)|^{2} d t .
\end{gathered}
$$

Combining inequalities (138), (144), and (149) with inequality (134), we obtain

$$
\begin{aligned}
\frac{1}{2}|w(s)|^{2} & +\frac{1}{4} \mu(0)\left|\int_{0}^{s} A^{1 / 2} w(\tau) d \tau\right|^{2} \\
\leq & 2 \int_{0}^{s} \frac{\left|\mu^{\prime}(t)\right|}{\mu_{0}}\left[\mu(0)\left|\int_{0}^{t} A^{1 / 2} w(\tau) d \tau\right|^{2}\right] d t \\
& +\left(5 S_{1}^{2 p}+5 N S_{2}\right) \int_{0}^{s} \frac{1}{2}|w(t)|^{2} d t, \quad 0 \leq s \leq s_{0} .
\end{aligned}
$$

This implies

$$
w(s)=0, \quad 0 \leq s \leq s_{0} .
$$

We will prove that $w^{\prime}\left(s_{0}\right)=0$. In fact as $w$ belongs to class (25) we have that $w^{\prime} \in C_{s}^{0}\left(\left[0, s_{0}\right] ; H\right)$; that is, $\left(w^{\prime}(t), \xi\right)$ is continuous on $\left[0, s_{0}\right]$ for all $\xi \in H$. Consider $\xi \in D(A)$. Then by $(128)_{1}$ we obtain

$$
\begin{aligned}
& \left\langle w^{\prime \prime}(t), \xi\right\rangle_{E^{\prime} \times E}+\langle A w(t), \xi\rangle_{E^{\prime} \times E}+|u(t)|^{2 p}(w(t), \xi) \\
& +\left(|u(t)|^{2 p}-|z(t)|^{2 p}\right)(z(t), \xi) \\
& =0, \quad \text { a.e. } t \in\left(0, s_{0}\right) .
\end{aligned}
$$

Integrating this equality on $\left[0, s_{0}\right]$ and using (151), we derive

$$
\left(w^{\prime}\left(s_{0}\right), \xi\right)+\int_{0}^{s_{0}}\left(|u(t)|^{2 p}-|z(t)|^{2 p}\right)(z(t), \xi) d t=0
$$

This, (146), and (151) give

$$
\begin{aligned}
\left|\left(w^{\prime}\left(s_{0}\right), \xi\right)\right| & \leq \int_{0}^{s_{0}}\left|\left(|u(t)|^{2 p}-|z(t)|^{2 p}\right)\|z(t)\| \xi\right| d t \\
& \leq \int_{0}^{1} N|w(t)\|z(t)\| \xi| d t=0 .
\end{aligned}
$$

Therefore, $\left(w^{\prime}\left(s_{0}\right), \xi\right)=0$ for all $\xi \in D(A)$. By density we obtain $\left(w^{\prime}\left(s_{0}\right), \xi\right)=0$ for all $\xi \in H$. Thus $w^{\prime}\left(s_{0}\right)=0$. Note that the constants $S_{1}, S_{2}$, and $N$ given, respectively, by (139) and (147) are independent of $0 \leq s \leq s_{0}$.

We apply similar arguments to the problem

$$
\begin{gathered}
w^{\prime \prime}+A w+|u|^{2 p} w+\left(|u|^{2 p}-|z|^{2 p}\right) z \\
=0 \quad \text { in } L^{\infty}\left(s_{0}, 2 s_{0} ; D\left(A^{-1 / 2}\right)\right), \\
w\left(s_{0}\right)=0, \quad w^{\prime}\left(s_{0}\right)=0
\end{gathered}
$$

and we obtain $w(s)=0$ for $s_{0} \leq s \leq 2 s_{0}$. After a finite number of steps we proof that $w(s)=0$ for $0 \leq s \leq T$.

When $\mu^{\prime}=0$, that is, $\mu(t)=\mu_{0}$, for all $t \in[0, T]$, expressions (144), (149), and similar arguments used to obtain the preceding result, allow us to deduce the uniqueness of solutions in this case.

\section{Applications}

Let $\Omega$ be an open bounded set of $\mathbb{R}^{n}$ with boundary $\Gamma$ of class $C^{\infty}$. Let $A=-\Delta$ be the self-adjoint operator defined by the triplet $\left\{H_{0}^{1}(\Omega), L^{2}(\Omega),((u, v))\right\}$, where $((u, v))$ denotes the usual scalar product of $H_{0}^{1}(\Omega)$. The norm of $L^{2}(\Omega)$ is denoted by $|u|$.

Lemma 11. Let $\theta \geq-1$ be a real number. Then, $D\left(A^{(1+\theta) / 2}\right)$ is contained in $H^{1+\theta}(\Omega)$ and

the embedding of $D\left(A^{(1+\theta) / 2}\right)$ in $H^{1+\theta}(\Omega)$ is continuous.

Proof. First we prove (156) when $(1+\theta) / 2=m, m$ a natural number. More precisely, we prove that if $u \in D\left(A^{m}\right)$, then $u \in H^{2 m}(\Omega)$ and

$$
\|u\|_{H^{2 m}(\Omega)} \leq C_{m}\left|A^{m} u\right| .
$$

To prove (157), we use the method of mathematical induction. Consider $m=1$ and $u \in D(A)$. Then, by the regularity of solutions of elliptic problems, we obtain $u \in H^{2}(\Omega)$ and

$$
\|u\|_{H^{2}(\Omega)} \leq C_{1}|A u| .
$$

Assume that (157) holds for $m=h$. Consider $u \in D\left(A^{h+1}\right)$. Then, $A u \in D\left(A^{h}\right)$. By induction hypothesis it follows that $A u \in H^{2 h}(\Omega)$ and

$$
\|A u\|_{H^{2 h}(\Omega)} \leq C_{h}\left|A^{h}(A u)\right|=\left|A^{h+1} u\right| .
$$

Consider the problem

$$
\begin{aligned}
A u=g & \text { in } \Omega, \\
u=0 & \text { on } \Gamma,
\end{aligned}
$$

where $g=A u$. As $g \in H^{2 h}(\Omega)$, by the regularity of solutions of elliptic problems, we have $u \in H^{2 h+2}(\Omega)$ and

$$
\|u\|_{H^{2 h+2}(\Omega)} \leq C^{*}\|A u\|_{H^{2 h}(\Omega)} .
$$


Inequalities (159) and (161) provide

$$
\|u\|_{H^{2 h+2}(\Omega)} \leq C^{*} C_{h}\left|A^{h+1} u\right|=C_{h+1}\left|A^{h+1} u\right| .
$$

This and (158) prove (157).

Next we use the interpolation of Hilbert spaces. Consider a natural number $m$ such that $2 m>1+\theta$. By results of intermediate spaces, we have

$$
\left[H^{2 m}(\Omega), L^{2}(\Omega)\right]_{\gamma}=H^{(1-\gamma) 2 m}(\Omega), \quad 0 \leq \gamma \leq 1 .
$$

(cf. Lions and Magenes [8]). We have by (157) that the injections

$$
\begin{gathered}
D\left(A^{m}\right) \longrightarrow H^{2 m}(\Omega), \\
D\left(A^{0}\right)=L^{2}(\Omega) \longrightarrow L^{2}(\Omega)
\end{gathered}
$$

are continuous. Then, by interpolation of Hilbert spaces, we have that the injection

$$
\left[D\left(A^{m}\right), L^{2}(\Omega)\right]_{\gamma} \longrightarrow\left[H^{2 m}(\Omega), L^{2}(\Omega)\right]_{\gamma}, \quad 0 \leq \gamma \leq 1
$$

is continuous. We choose $\gamma_{0}=1-(1+\theta) / 2 m$. Then, by (163), we obtain

$$
\left[H^{2 m}(\Omega), L^{2}(\Omega)\right]_{\gamma_{0}}=H^{1+\theta}(\Omega) .
$$

Also

$$
\left[D\left(A^{m}\right), L^{2}(\Omega)\right]_{\gamma_{0}}=D\left(A^{(1+\theta) / 2}\right) .
$$

These last two equalities and (165) give the lemma.

Consider the operator $\delta\left(x-x_{0}\right), x_{0} \in \Omega$. For $\theta>(n / 2)-1$; we have that

$$
H^{1+\theta}(\Omega) \hookrightarrow C^{0}(\bar{\Omega})
$$

This embedding and (156) imply that

$$
\begin{aligned}
& \text { the embedding of } D\left(\mathrm{~A}^{(1+\theta) / 2}\right) \text { in } C^{0}(\bar{\Omega}) \\
& \text { is continuous }\left(\theta>\frac{n}{2}-1\right) .
\end{aligned}
$$

Define

$$
\left\langle\delta\left(x-x_{0}\right), \varphi\right\rangle=\varphi\left(x_{0}\right), \quad \varphi \in D\left(A^{(1+\theta) / 2}\right) .
$$

By (169) we have that $\delta\left(x-x_{0}\right) \in D\left(A^{-(1+\theta) / 2}\right)$. Thus, for

$$
\begin{gathered}
f=v \delta\left(x-x_{0}\right) \quad \text { with } v \in W^{1,1}(0, T), \\
\delta\left(x-x_{0}\right) \in D\left(A^{-(1+\theta) / 2}\right) \quad\left(\theta>\frac{n}{2}-1\right),
\end{gathered}
$$

Theorems 1 and 3 give, respectively, solutions $u$ of problems (14) and (22).
Consider (171) for the particular case $\theta=0$. Then Theorems 1 and 6 provide a unique solution $u$ of the problem

$$
\begin{aligned}
u_{t t}-\mu u_{x x}+\left(\int_{a}^{b}|u|^{2} d x\right)^{p} u & \\
= & v \delta\left(x-x_{0}\right) \quad \text { in }(a, b) \times(0, T) \quad(p \geq 1) ; \\
& u(a, t)=u(b, t)=0, \quad t \in(0, T) ; \\
u(x, 0) & =u^{0}(x), \quad u_{t}(x, 0)=u^{1}(x), \quad x \in(a, b) .
\end{aligned}
$$

On the other hand, we have $X=L^{2}(\Omega)$ if $\lambda=0$ ( $X$ defined in (21)). In this case $-\theta / 2 \leq 0<(1-\theta) / 2$, that is, $0 \leq \theta<1$. The two restrictions $\theta>n / 2-1$ and $0 \leq \theta<1$ give, respectively, for $n=1,2$, and 3 the variations $0 \leq \theta<1,0<\theta<1$, and $1 / 2<\theta<1$. In all three cases, Corollary 5 gives a solution $u$ of the problem

$$
\begin{aligned}
& u^{\prime \prime}-\mu \Delta u+\left[\int_{0}^{T}\left(\int_{\Omega}|u|^{2} d x\right)^{p / 2} d t\right] u \\
& =v \delta\left(x-x_{0}\right) \quad \text { in } \Omega \times(0, T) \quad(p \geq 1) ; \\
& u=0 \quad \text { in } \Gamma \times(0, T) ; \\
& u(x, 0)=u^{0}(x), \quad u^{\prime}(x, 0)=u^{1}(x), \quad x \in \Omega,
\end{aligned}
$$

where $\Omega$ is an open bounded set of $\mathbb{R}^{n}, n=1,2,3$.

\section{Conflict of Interests}

The authors report that there is no conflict of interests in the publication of this paper.

\section{References}

[1] J.-L. Lions, Équations aux Dérivées Partielles-Interpolation. Vol. I, EDP Sciences, Les Ulis, Paris, France, 2003, Oeuvres choisies de Jacques Louis Lions, 2003.

[2] J.-L. Lions, Some Methods in the Mathematical Analysis of System and Their Control, Science Press, Beijing, China; Gordon Breach, Science Publishers, New York, NY, USA, 1981.

[3] C. Grotta Ragazzo, "Chaos and integrability in a nonlinear wave equation," Journal of Dynamics and Differential Equations, vol. 6, no. 1, pp. 227-244, 1994.

[4] L. I. Schiff, "Nonlinear meson theory of nuclear forces. I. Neutral scalar mesons with point-contact repulsion," The Physical Reviews, vol. 84, no. 1, pp. 1-9, 1951.

[5] K. Jörgens, "Des aufangswert problem in grossen für eine klasse nichtlinearer wellengleichungen," Mathematische Zeitschrift, vol. 77, pp. 295-308, 1971.

[6] A. T. Lourêdo, M. A. F. Araújo, and M. Milla Miranda, "On a nonlinear wave equation with boundary damping," Mathematical Methods in the Applied Sciences, 2013.

[7] A. Pazy, Semigroups of Linear Operators and Applications to Partial Differential Equations, vol. 44, Springer, New York, NY, USA, 1993.

[8] J. L. Lions and E. Magenes, Problèmes Aux Limites NonHomogènes et Applications. Vol. I, Dunod, Paris, France, 1968. 
[9] H. Brezis, Functional Analysis, Sobolev Spaces and Partial Differential Equations, Springer, New York, NY, USA, 2010.

[10] V. Komornik, Exact Controllability and Stabilization. The Multiplier Method, John Wiley and Sons, Paris, France, 1994.

[11] L. A. Medeiros and M. Milla Miranda, Espaços de Sobolev (Iniciação aos Problemas Elíticos Não-Homogêneos), IM-UFRJ, Rio de Janeiro, Brazil, 5th edition, 2006.

[12] J. L. Lions, Quelques Méthodes De Résolution des Problémes aux Limites Non-Linéaires, Dunod, Paris, France, 1969.

[13] A. Vicente and C. L. Frota, "On a mixed problem with a nonlinear acoustic boundary condition for a non-locally reacting boundaries," Journal of Mathematical Analysis and Applications, vol. 407, no. 2, pp. 328-338, 2013.

[14] J. Simon, "Compact sets in the space $L^{p}(0, T ; B)$," Annali di Matematica Pura ed Applicata, vol. 146, pp. 65-96, 1987. 


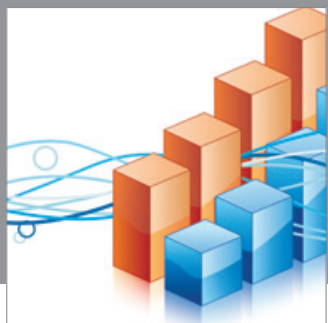

Advances in

Operations Research

mansans

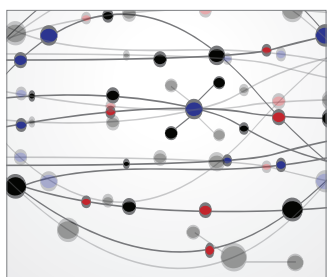

The Scientific World Journal
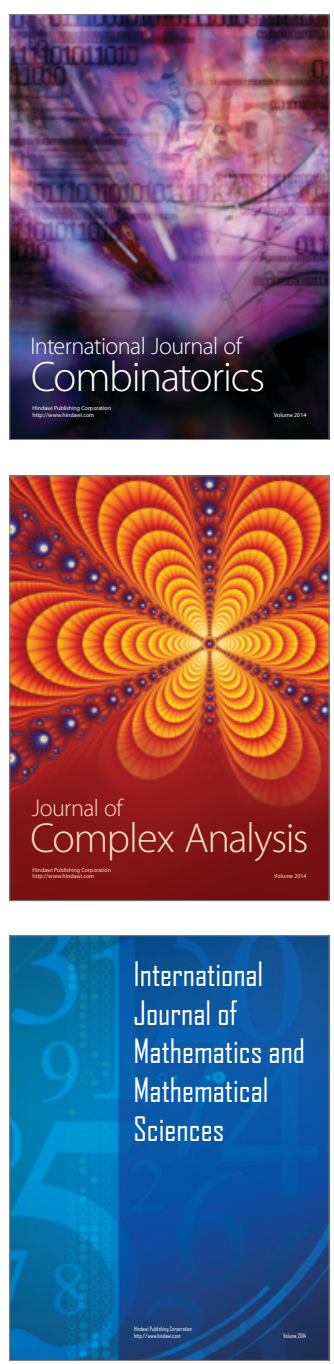
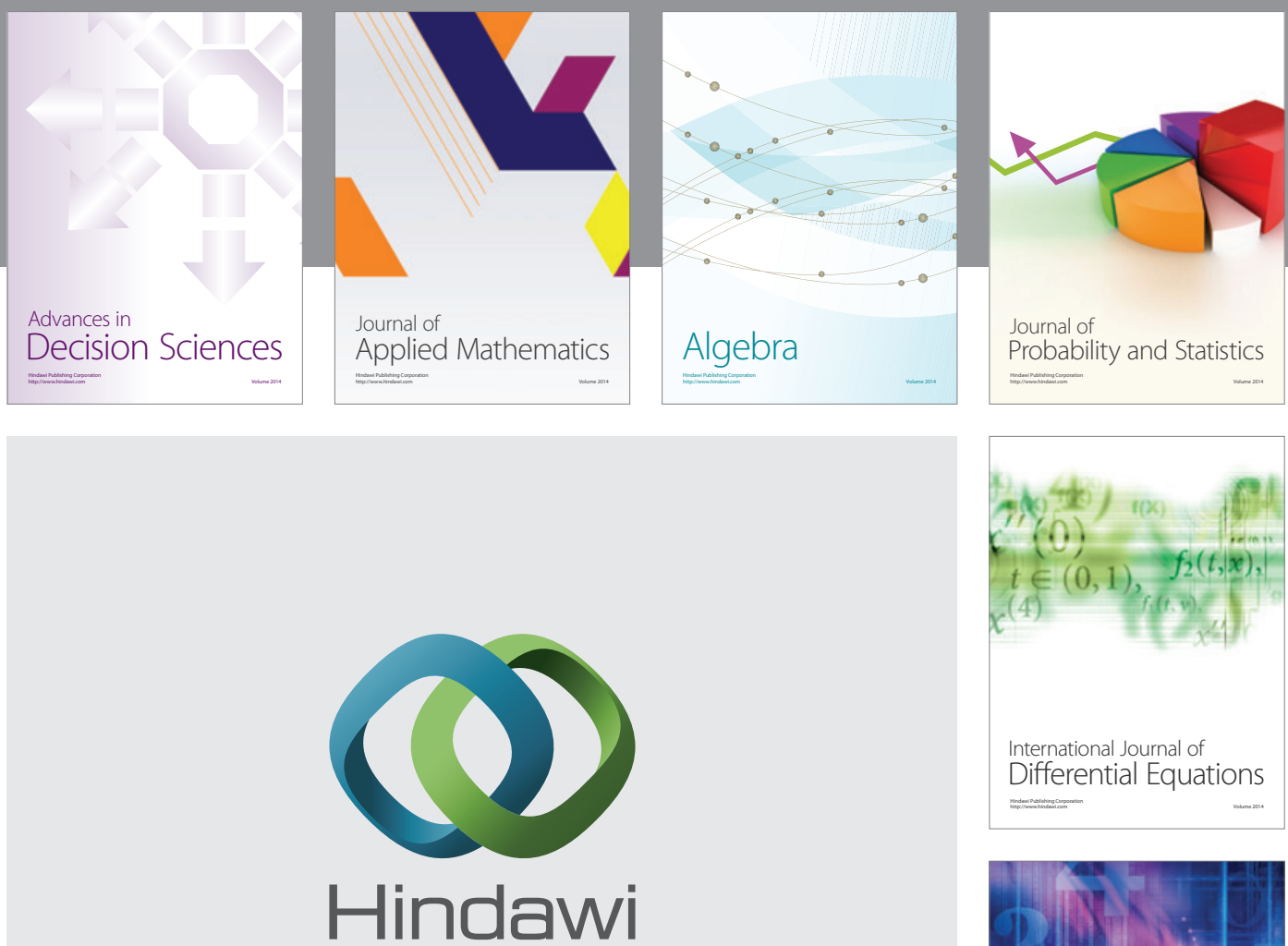

Submit your manuscripts at http://www.hindawi.com
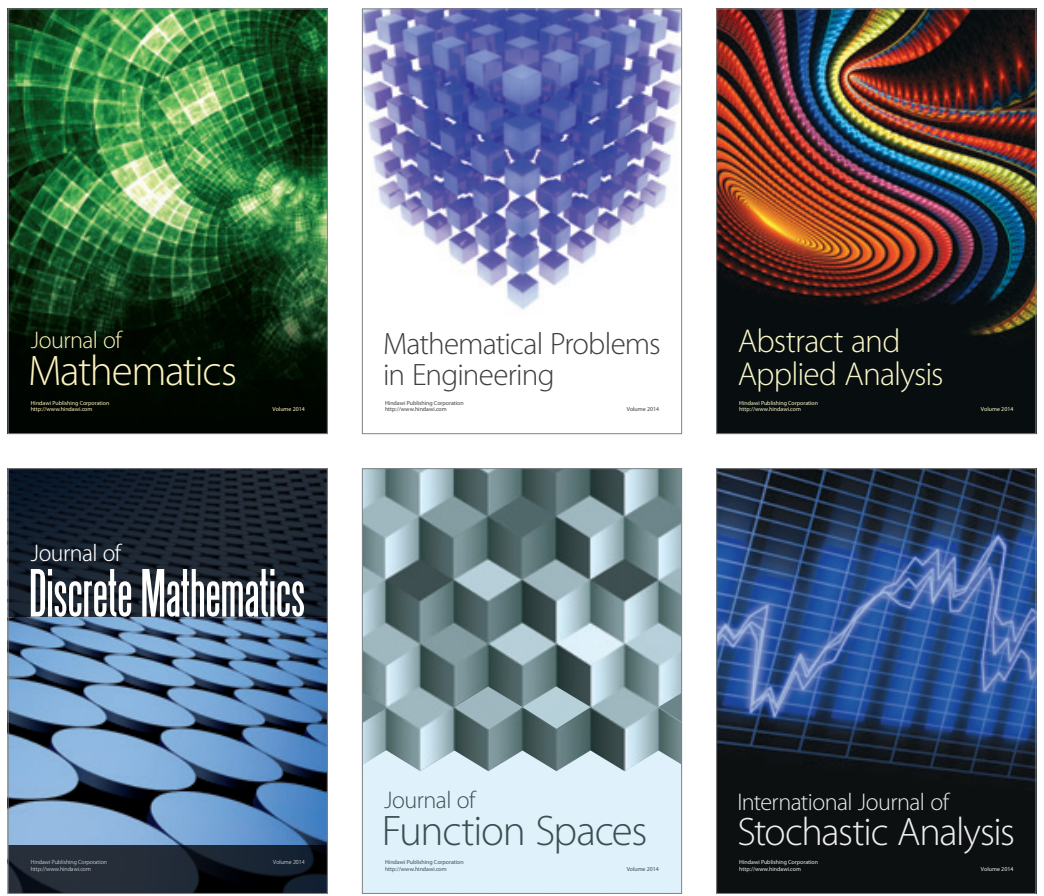

Journal of

Function Spaces

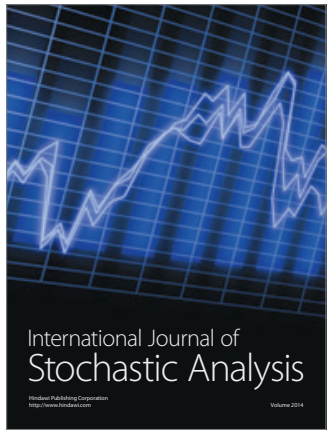

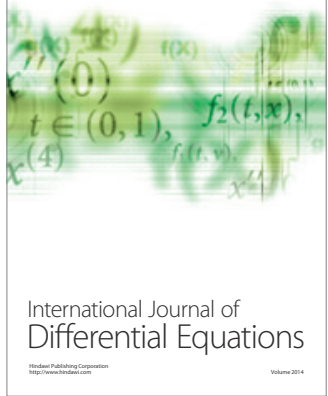
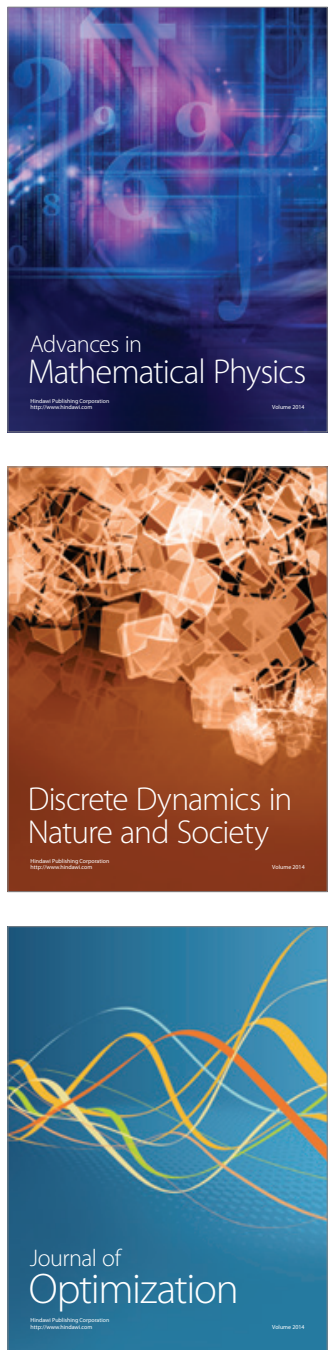